\title{
Bone height measurement of maxillary and mandibular bones in panoramic radiographs of edentulous patients
}

\author{
Çağrı Ural ${ }^{1}$, Cihan Bereket ${ }^{2}$, İsmail Şener ${ }^{2}$, Ali Murat Aktan ${ }^{3}$, Yusuf Ziya Akpinar ${ }^{4}$ \\ ${ }^{1}$ Assistant Professor, Department of Prosthodontics, Faculty of Dentistry, Ondokuz Mayis University, Samsun, Turkey. \\ ${ }^{2}$ Assistant Professor, Department of Oral and Maxillofacial Surgery, Faculty of Dentistry, Ondokuz Mayis University, Samsun, \\ Turkey. \\ ${ }^{3}$ Assistant Professor, Department of Oral Diagnosis and Radiology, Faculty of Dentistry, Gaziantep University, Gaziantep, Tur- \\ key. \\ ${ }^{4}$ Private Practise, Samsun, Turkey.
}

Correspondence:

Ondokuz Mayls University

Faculty of Dentistry Department of Prosthodontics

55139 Kurupelit- SAMSUN - TURKEY

E-mail:cagriural@omu.edu.tr

Received: $25 / 08 / 2010$ Accepted: $17 / 12 / 2010$
Ural Ç, Bereket C, Şener İ, Aktan AM, Akpinar YZ. Bone height measurement of maxillary and mandibular bones in panoramic radiographs of edentulous patients. J Clin Exp Dent. 2011;3(1):e5-9..

http://www.medicinaoral.com/odo/volumenes/v3i1/jcedv3ilp5.pdf

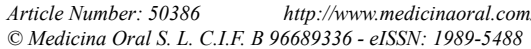

\begin{abstract}
Objectives. The purpose of this in vitro study were to determine variation in maxillary and mandibular vertical measurements and to assess vertical bone loss made from panoramic radiographs in edentulous measurements.

Study Design. In this descriptive study, a total of 600 panoramic radiograph's of edentulous patient were examined. The mean ages of edentulous patients were 31 and 87 years respectively. Measurements were made from reference lines drawn from anatomic landmarks on standardized panoramic rediographs. All radiographs were made using a standardized manner by the same technician. Twelve sites were measured on every panoramic radiograph whenever possible, five sites in the maxilla and seven sites in the mandible.

Results. In the maxilla all vertical measurements distance were significantly greater in the edentulous men than in the edentulous women $(p<0,05)$. In the mandible all vertical measurements distance were significantly greater in the edentulous men than in the edentulous women $(\mathrm{p}<0,05)$.

Conclusion. The results of this study may guide clinicians to make primer decision of implant insertion area for implant supported prosthesis in edentulous patients
\end{abstract}

Key words: Panoramic radiograph, alveolar bone resorption, edentulous, vertical height. 


\section{Introduction}

The goal of modern dentistry is to restore the patient to normal contour, function, comfort, aesthetics, speech and health, regardless of the atrophy, disease or injury of the stomatognatic system. The loss of teeth causes remodeling and resorption of the surrounding alveolar bone and eventually leads to atrophic edentulous ridges. In edentulous patients, reduction of the residual ridge is one of the most important factors affecting denture support, retention, stability and masticatory function (1). Resorbtion of the residual ridge has been estimated with various radiographic techniques: lateral cephalometric radiographs (2) and panoramic radiographs (3-6).

The use of dental implants to provide support for prosthesis offers a multitude of advantages compared with the use of removable soft tissue-born restorations. Osseointegrated implant treatment has increased over the past decade, with functional 5-year success rate of $90 \%$ or higher (7). One of the factors that contribute to this successful therapy is the careful surgical and prosthetic treatment plan. The main components of this plan are diagnostic waxing, study of dental cast, and clinical and radiographic examinations (8). Therefore, the ability to determine the quality and quantity of available bone in the potential implant site is paramount importance.

Treatment planning for dental implant patients is often complicated by the unknown curve of anatomical structures. The presence of a neurovascular bundle in the interforaminal region may present some risks during the implant surgery and therefore preoperative identification is considered importance (9). Regardless of the type of the implant system, preoperative assessment requires various radiographic examinations, including basic plain radiography as well as advanced methods such as computed tomography (CT), (10) and also panoramic radiography is commonly used in large institutional practices as the sole method of screening edentulous and dentate patients (11) and it is extremely important in diagnosis and treatment of many oral and maxillofacial diseases. However a panoramic radiography is a two dimensional image, lacking information in the bucco-lingual direction and magnification in both vertical and horizontal directions. It has been shown that cross-sectional imaging (CT) (12-14) alternative for the precise visualization of the anatomical structures but the economic and technical properties has to be used the panoramic radiographs (15).

The image quality of the panoramic radiography is increased by the digital panoramic radiography. It is a fact that panoramic imaging is widely used for evaluation of the jaws for planning implant surgery and the other purposes. In studies of residual ridge resorption, problems due to distortion of images, variation in the magnification factor at different object depths, and difficulty in standardizing the head position among various patients limit the use of the panoramic radiograph (16).

The purpose of this study was to determine vertical height measurements by using digital panoramic radiographs in Turkish population, and to assess the amount of residual ridge resorbtion in the midline, premolar and molar region which is important for the implant treatment planning.

\section{Material and Methods}

In this descriptive study, panoramic radiographs of edentulous patients in the Ondokuz Mayis University, dental faculty archives were selected using a simple method and studied. The only selection criteria were that the radiographs be of good quality. A total of 650 digital panoramic radiographs were obtained with the panoramic cephalometric x-ray unit (Gendex Orthoralix ${ }^{\circledR}$ 9200 DDE, USA). All images were taken by the same

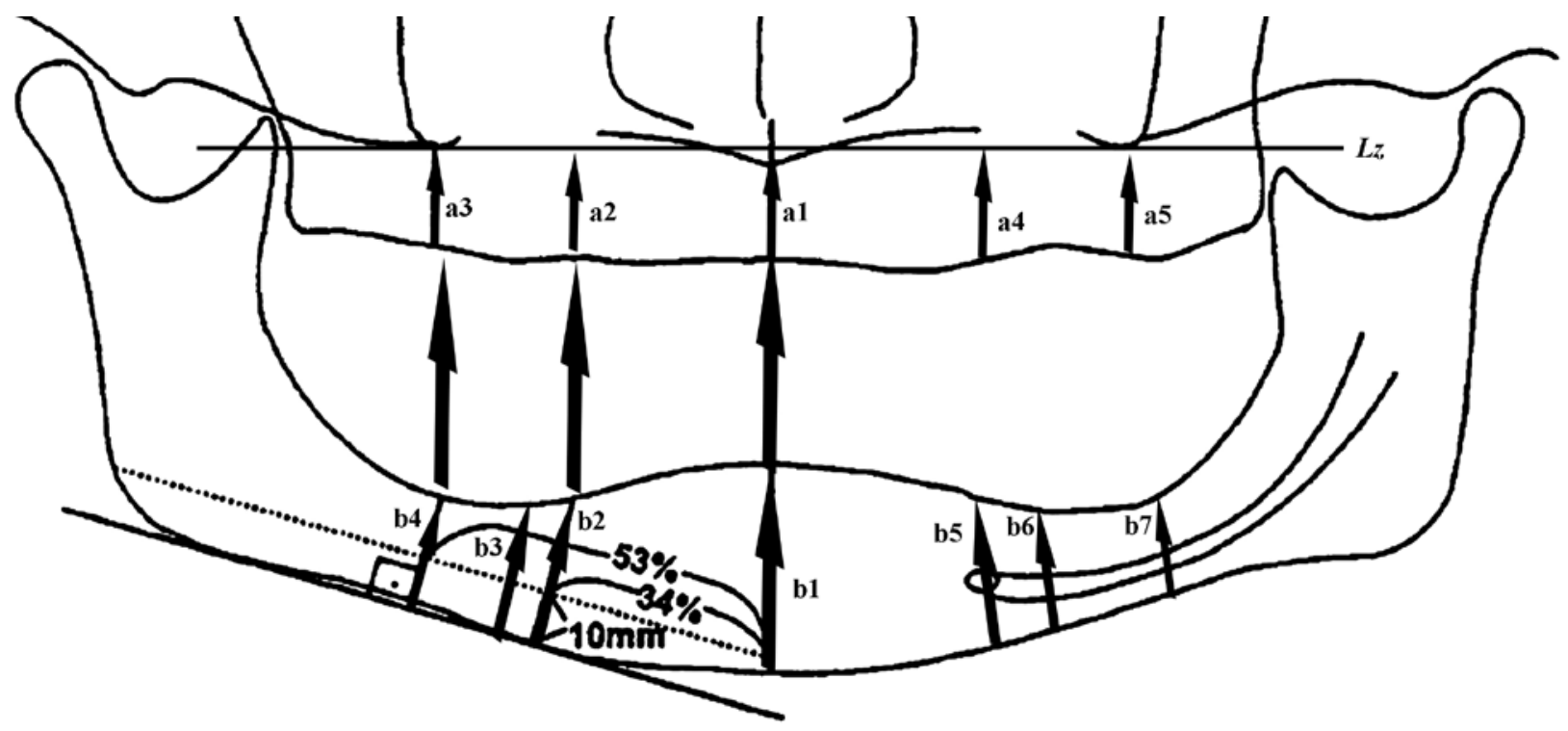

Fig. 1. Reference lines and measuring points in mandible and maxilla. 
technician according to a standardized protocol for patient positioning and exposure parameter setting $(70 \mathrm{kV}$, 8-10 mA, 15-19 sn).

Images were obtained from 650 edentulous patient (273 male - 377 female) which were attended the Department of Prosthodontics, Faculty of Dentistry, Ondokuz Mayis University. Fifty radiograph were removed from the study. The subjects were aged between 31-87 years of age. The magnification ratio of the panoramic machine was 1:1.25. Radiograph's criteria selection were: some anatomic landmarks such as the inferior and posterior border of the mandible, the most inferior border of both orbita, the most inferior margins of the zygomatic process of the maxilla, mental foramen and the infraorbital foramen must be distinct on at least one side and there must be no distortion. Panoramic radiograph did not meet these criteria and were excluded. Xie et al. (16) stated that, the first premolar and the first molar were located approximately 34 and $53 \%$, respectively of the length of the mandibular body from the midline (Fig. 1). These proportions were used to determine of mandibular first premolar and molar area on the edentulous patients' radiograph.

Twelve sites were measured on each radiograph. A line joined the inferior margins of the zygomatic process of the maxilla (Lz). The midline was determined by images of the nasal septum anterior nasal spine and nasopalatine foramen (Fig. 1).

The measurements a1 (midline), a2-a4 (Premolar region), a3-a5 (Molar region) represented vertical distances from line Lz to the alveolar crest. In the mandible, a line was drawn tangential to the most inferior points at the mandibular angle and the lower border of the mandi- ble. The measurement b1 (Midline), b2-b5 (Mental foramen region), b3-b6 (premolar region) and b4-b7 (molar region).

Each radiograph was viewed digitally. One investigator was responsible for selection of the panoramic radiographs and performing the measurements. The measurements were made digitally according to magnification ratio on an analyses programe (Gendex, VixWin Platinum v1.1, Germany). In order to examine the effect of gender the statistical analysis of differences between men and women was performed with Student t-test. The $5 \%$ significance level was used for statistically significance.

\section{Results}

All the vertical bone height measurements and standard deviations were listed on Table 1. Of the 600 panoramic radiographs, 327 (58\%) were of women and 273 (42\%) were of men. One thousand two hundred alveolar ridges were investigated. The mean age of the subjects was 3185 years; the age ranges were $31-85$ years in women and 31-87 years in men.

In the maxilla all vertical measurements distance were significantly greater in the edentulous men than in the edentulous women $(\mathrm{p}<0,05)$. In the mandible all vertical measurements distance were significantly greater in the edentulous men than in the edentulous women $(\mathrm{p}<0,05)$.

\section{Discussion}

In this study the bone height measurement affected from gender of the population. Alveolar atrophy after the removal of teeth and the presence of tooth components in

\begin{tabular}{|c|c|c|c|c|c|c|}
\hline & \multicolumn{3}{|c|}{ Men } & \multicolumn{3}{c|}{ Women } \\
\cline { 2 - 7 } & Mean & Min-Max & s.d. & Mean & Min-Max & s.d. \\
\hline $\boldsymbol{a}_{\boldsymbol{1}}$ & 14,94 & $5,30-27,70$ & $\pm 3,91$ & 13,81 & $5,10-24,75$ & $\pm 4,25$ \\
\hline $\boldsymbol{a}_{\boldsymbol{2}}$ & 15,32 & $9,55-21,70$ & $\pm 3,13$ & 14,41 & $7,60-27,15$ & $\pm 4,05$ \\
\hline $\boldsymbol{a}_{\mathbf{3}}$ & 14,75 & $9,35-24,85$ & $\pm 3,14$ & 14,26 & $7,05-24,50$ & $\pm 3,34$ \\
\hline $\boldsymbol{a}_{\boldsymbol{4}}$ & 14,59 & $7,40-23,80$ & $\pm 3,68$ & 13,48 & $4,25-20,65$ & $\pm 3,71$ \\
\hline $\boldsymbol{a}_{\boldsymbol{5}}$ & 14,31 & $6,55-21,70$ & $\pm 3,36$ & 13,43 & $5,30-21,20$ & $\pm 3,86$ \\
\hline $\boldsymbol{b}_{\boldsymbol{1}}$ & 24,86 & $10,65-38,45$ & $\pm 5,64$ & 21,50 & $11,45-33,80$ & $\pm 4,69$ \\
\hline $\boldsymbol{b}_{\mathbf{2}}$ & 24,26 & $10,60-37,05$ & $\pm 5,73$ & 20,08 & $9,95-33,30$ & $\pm 5,56$ \\
\hline $\boldsymbol{b 3}$ & 23,50 & $10,40-36,75$ & $\pm 6,04$ & 20,28 & $10,05-31,55$ & $\pm 5,36$ \\
\hline $\boldsymbol{b} 4$ & 21,86 & $10,45-34,50$ & $\pm 6,01$ & 17,56 & $6,75-29,60$ & $\pm 5,66$ \\
\hline $\boldsymbol{b 5}$ & 20,10 & $11,20-32,45$ & $\pm 4,77$ & 16,92 & $6,65-28,45$ & $\pm 4,66$ \\
\hline $\boldsymbol{b} 6$ & 22,08 & $10,60-32,50$ & $\pm 5,56$ & 18,68 & $7,00-31,10$ & $\pm 5,56$ \\
\hline $\boldsymbol{b} 7$ & 20,20 & $10,75-29,60$ & $\pm 4,36$ & 16,47 & $4,25-28,60$ & $\pm 4,88$ \\
\hline
\end{tabular}

Table 1. Mean standard deviations and min-max values of measurements value (mm) in women and men population 
both the upper and lower jaws of edentulous patients have always been topics of discussion for dentists (17). This study attempted to test the use fullness of panoramic radiography in assessing bone height for implant recipient sites.

Panoramic radiography is widely used and often a tool used in routine dental diagnosis and prognosis. Especially implant treatment planning the panoramic radiographs are the most important criteria for implant selection. Assessing residual ridge resorption, position of maxillary sinus, mandibular canal and mental foramen with panoramic radiographs is practical for examinations of large samples of patients (16). A previous study posed that the use of landmarks in the edentulous mandible is reliable as a reference point in clinical studies (18). Larheim and Svanaes (19) investigated the precision of measurements of mandibular linear dimensions in panoramic radiographs and found that the variability of vertical measurements made from repeated panoramic radiographs is small when patients are properly positioned in the panoramic machine. According to Xie et al. (20) , if reference lines and measuring points are located in the same vertical plane, variations in vertical measurements in the mandible and the posterior regions of the maxilla fall within a small range. In a study (19) of the reproducibility of panoramic radiographs were exposed under different clinical radiographic conditions. The method error for assessment of vertical distances in the mandible fell mostly within $3 \%$ of total variance. Every distance value in the radiographic films was significantly longer than in the direct measurement, despite considering the magnification rate. In the present study magnification, factor was not necessary to correct the radiographic heights, because all radiographs were taken the same panoramic apparatus.

Almost all previous studies showed that the most common horizontal location of the mental foramen was aligned with the second premolar (21-23), which was followed by being located between the first and second premolars (24). Dry skulls or radiographic films were used in most of the previous studies, so they could use the center of the mental foramen as a reference point. Wang et al . (22) reported that the average distance between the bottom of the lower second premolar socket and the superior border of the mental foramen is 2.50 $\mathrm{mm}$. When the literature evaluated previous studies $(16,25)$ reported that according to results dentate subjects, the first premolar and the first molar were located approximately 34 and $53 \%$, respectively, of the length of the mandibular body from the midline so we made our measurements in the premolar and molar region according to this percentage.

Vertical measurements that obtained in present study, significant differences were found in the heights of the mandible and maxilla between edentulous subject of both sexes. In our study the decrease in the height of the edentulous mandible was more pronounced in women than in men. Ortman et al. (26) and Baat et al. (27) reported similar results. In postmenopausal women, deficiency of estrogen hormone accelerates skeletal bone loss and may result in rapid alveolar bone resorption (28). However this difference between the genders was not obvious in the maxilla. The explanation may lie in different bone quantities between the mandible and maxilla; trabecular bone is more often looser in the edentulous maxilla than mandible.

There is limited information in the literature about the bone heights of edentulous patients. Most of the literature compared dentate and edentulous patients. In the present study, only edentulous patient's measurements were evaluated.

The measurement value of the maxilla at the anterior, first premolar and first molar regions were significantly greater in men than women. Residual ridge resorption is usually more rapid in the premolar and molar region than the anterior region of the mandible because of the lower position of the reversal line in the posterior region (29). The reversal line on the lingual side occurs along the mylohyoid ridge, which is the limit between the resorptive alveolar field and depository field of the basal bone (29). In addition, the inner side aspect of the anterior area of the mandible provides attachment for the genial muscles (16). These attachments, because of the functional forces of the genial muscles, probably protect this area from extreme alveolar bone loss and reduction in vertical height.

Vertical heights of alveolar bone, localization of anatomic landmarks are important factors for planning of the implant supported prosthesis. The results of this study may guide clinicians to make primer decision of implant insertion area for implant supported prosthesis in edentulous patients.

\section{References}

1. Soikkonen K, Ainamo A, Xie Q. Height of the residual ridge and radiographic appearance of bony structure in the jaws of clinically edentulous elderly people. J Oral Rehabil. 1996;23: 470-5.

2. Jonkman RE, van Waas MA, Plooij J, Kalk W. Measuring mandibular ridge reduction on oblique cephalometric radiographs. J Craniomaxillofac Surg. 1991;19: 27-30.

3. van Waas MA. Ridge resorption in denture wearers after vestibuloplasty and lowering of the floor of the mouth, measured on panoramic radiographs. Dentomaxillofac Radiol. 1983;12: 115-21.

4. Swart JG, Allard RH. Subperiosteal onlay augmentation of the mandible: a clinical and radiographic survey. J Oral Maxillofac Surg. 1985;43: 183-7.

5. Wilding RJ, Levin I, Pepper R. The use of panoramic radiographs to measure alveolar bone areas. J Oral Rehabil. 1987;14: 557-67.

6. Humphries S, Devlin H, Worthington H. A radiographic investigation into bone resorption of mandibular alveolar bone in elderly edentulous adults. J Dent. 1989;17: 94-6.

7. Adell R, Lekholm U, Rockler B, Branemark PI. A 15-year study of osseointegrated implants in the treatment of the edentulous jaw. Int J Oral Surg. 1981;10: 387-416.

8. Brånemark PI, Hansson $\mathrm{BO}$, Adell R, Breine U, Lindström J, 
Hallén O, et al. Osseointegrated implants in the treatment of the edentulous jaw. Experience from a 10-year period. Scand J Plast Reconstr Surg Suppl. 1977;16:1-132.

9. Sakakura CE, Loffredo Lde C, Scaf G. Diagnostic agreement of conventional and inverted scanned panoramic radiographs in the detection of the mandibular canal and the mental foramen. J Oral Implantol. 2004;30: 2-6.

10. Jacobs R, Adriansens A, Naert I, Quirynen M, Hermans R, Van Steenberghe D. Predictability of reformatted computed tomography for pre-operative planning of endosseous implants. Dentomaxillofac Radiol. 1999;28: 37-41.

11. Scandrett FR, Tebo HG, Miller JT, Quigley MB. Radiographic examination of the edentulous patient. I. Review of the literature and preliminary report comparing three methods. Oral Surg Oral Med Oral Pathol. 1973;35: 266-74.

12. Jacobs R, Mraiwa N, Van Steenberghe D, Sanderink G, Quirynen M. Appearance of the mandibular incisive canal on panoramic radiographs. Surg Radiol Anat. 2004;26: 329-33.

13. Dharmar S. Locating the mandibular canal in panoramic radiographs. Int J Oral Maxillofac Implants. 1997;12: 113-7.

14. Bou Serhal C, Jacobs R, Flygare L, Quirynen M, van Steenberghe D. Perioperative validation of localisation of the mental foramen. Dentomaxillofac Radiol. 2002;31: 39-43.

15. Thanyakarn C, Hansen K, Rohlin M. Measurements of tooth length in panoramic radiographs. 2: Observer performance. Dentomaxi1lofac Radiol. 1992;21: 31-5.

16. Xie Q, Wolf J, Ainamo A. Quantitative assessment of vertical heights of maxillary and mandibular bones in panoramic radiographs of elderly dentate and edentulous subjects. Acta Odontol Scand. 1997;55: 155-61.

17. Yamaoka M, Furusawa K, Fujimoto K, Uematsu T. Completely impacted teeth in dentate and edentulous jaws. Aust Dent J. 1996;41: $169-72$.

18. Batenburg RH, Stellingsma K, Raghoebar GM, Vissink A. Bone height measurements on panoramic radiographs: the effect of shape and position of edentulous mandibles. Oral Surg Oral Med Oral Pathol Oral Radiol Endod. 1997;84: 430-5.

19. Larheim TA, Svanaes DB. Reproducibility of rotational panoramic radiography: mandibular linear dimensions and angles. Am J Orthod Dentofacial Orthop. 1986;90: 45-51.

20. Xie Q, Soikkonen K, Wolf J, Mattila K, Gong M, Ainamo A. Effect of head positioning in panoramic radiography on vertical measurements: an in vitro study. Dentomaxillofac Radiol. 1996;25: 61-6.

21. Phillips JL, Weller RN, Kulild JC. The mental foramen: 1. Size, orientation, and positional relationship to the mandibular second premolar. J Endod. 1990;16: 221-3.

22. Wang TM, Shih C, Liu JC, Kuo KJ. A clinical and anatomical study of the location of the mental foramen in adult Chinese mandibles. Acta Anat (Basel). 1986;126: 29-33.

23. al Jasser NM, Nwoku AL. Radiographic study of the mental foramen in a selected Saudi population. Dentomaxillofac Radiol. 1998;27: 341-3.

24. Kim IS, Kim SG, Kim YK, Kim JD. Position of the mental foramen in a Korean population: a clinical and radiographic study. Implant Dent. 2006;15: 404-11.

25. Saglam AA. The vertical heights of maxillary and mandibular bones in panoramic radiographs of dentate and edentulous subjects. Quintessence Int. 2002;33: 433-8.

26. Ortman LF, Hausmann E, Dunford RG. Skeletal osteopenia and residual ridge resorption. J Prosthet Dent. 1989;61: 321-5.

27. de Baat C, Kalk W, van 't Hof M. Factors connected with alveolar bone resorption among institutionalized elderly people. Community Dent Oral Epidemiol. 1993;21: 317-20.

28. Devlin H, Ferguson MW. Alveolar ridge resorption and mandibular atrophy. A review of the role of local and systemic factors. Br Dent J. 1991;170: 101-4.

29. Mercier P. Ridge reconstruction with hydroxylapatite. Part 1. Anatomy of the residual ridge. Oral Surg Oral Med Oral Pathol. 1988;65: 505-10. 